\section{THE USEFULNESS OF COMPENSATION STATISTICS TO DETECT HEAT-RELATED HEALTH OUTCOMES IN A TEMPERATE CLIMATE: THE EXPERIENCE OF QUEBEC}

${ }^{1,2}$ France Labrèche, ${ }^{2}$ Ariane Adam-Poupart, ${ }^{1}$ Marc-Antoine Busque, ${ }^{1}$ Patrice Duguay, ${ }^{3}$ Michel Fournier, 1,2Joseph Zayed, 2,4Audrey Smargiassi. 'Institut de Recherche Robert-Sauvé en Santé Et Sécurité Du Travail (IRSST), Montreal, Quebec, Canada; ${ }^{2}$ Department of Environmental and Occupational Health, School of Public Health, Université de Montréal, Montreal, Quebec, Canada; ${ }^{3}$ Direction de Santé Publique, Agence de La Santé Et Des Services Sociaux de Montréal, Montreal, Quebec, Canada; ${ }^{4}$ Institut National de Santé Publique Du Québec (INSPQ), Montreal, Quebec, Canada

\subsection{6/oemed-2014-102362.123}

Objectives To explore relationships between summer outdoor temperatures in Quebec (Canada) and occupational compensation statistics for heat-related illnesses.

Method Daily compensation counts of heat-related illnesses (heat strain, heatstroke, loss of consciousness, etc.), occurring between May and September, were obtained from the workers' compensation board of Quebec for each health region between 1998 and 2010. Regional daily maximum outdoor temperatures were obtained from Environment Canada. Associations between daily compensation counts and temperature were estimated using negative binomial or Poisson regression models for each region and were adjusted for relative humidity and temporal trends. Pooled effect sizes for Quebec (all health regions combined) were obtained using a fixed effect model for meta-analysis.

Results In an average population of 3.7 million workers, 259 illnesses classified as heat-related were compensated between 1998 and 2010, giving an average annual rate of 0.11 case per 100000 workers per summer month. During the study period, $63.0 \%$ of heat-related outcomes occurred on days with a maximum daily average temperature below $30^{\circ} \mathrm{C}$. Occupations with the largest number of compensations were those of labourers (32\%), firefighters (11\%) and truck drivers (4\%). The pooled incidence rate ratio (IRR) was $1.41(95 \%$ CI $1.35-1.46)$ per $1^{\circ} \mathrm{C}$ increase in daily maximum temperature. Effects of barometric pressure and lag will be explored.

Conclusions Heat-related illnesses do occur in temperate climates. Our results suggest that compensation statistics, albeit crude indicators of health effects, can be useful to identify industry sectors and occupations that would benefit from preventive interventions aimed at high risk workers.

\section{WORKPLACE PSYCHOSOCIAL RISK FACTORS FOR CARPAL TUNNEL SYNDROME: A POOLED PROSPECTIVE STUDY}

${ }^{1,2}$ Carisa Harris Adamson, ${ }^{1}$ Ellen Eisen, ${ }^{3}$ Kurt Hegman, ${ }^{3}$ Matthew Thiese, ${ }^{4}$ Barbara Silverstein, ${ }^{4}$ Stephen Bao, ${ }^{5}$ Arun Garg, ${ }^{5}$ Jay Kapellusch, ${ }^{6}$ Susan Burt, ${ }^{7}$ Fred Gerr, ${ }^{7}$ Linda Merlino, ${ }^{8}$ Ann Marie Dale, ${ }^{8}$ Bradley Evanoff, ${ }^{1,9}$ David Rempel. ${ }^{1}$ University of California, Berkeley, Berkeley, CA, USA; ${ }^{2}$ Samuel Merritt University, Oakland, CA, USA; ${ }^{3}$ University of Utah, Salt Lake City, UT, USA; ${ }^{4}$ Washington State Department of Labor and Industries, Safety and Health Assessment and Research for Prevention (SHARP) Program, Tumwater, WA, USA; ${ }^{5}$ University of Wisconsin-Milwaukee, Milwaukee, WI, USA; ${ }^{6}$ National Institute for Occupational Safety and Health, Atlanta, GA, USA; ${ }^{7}$ University of lowa, lowa City, IA, USA; ${ }^{8}$ Washington University School of Medicine, St Louis, MO, USA; ${ }^{9}$ University of California, San Francisco, San Francisco, CA, USA

\subsection{6/oemed-2014-102362.124}

Objectives Seven research groups conducted coordinated studies of carpal tunnel syndrome (CTS). In this analysis of the pooled cohort, we estimate associations of workplace psychosocial factors and CTS incidence with adjustment for biomechanical factors.
Method 3515 workers were followed up to 7 years. Case criteria included symptoms consistent with CTS and an abnormal electrodiagnostic study. Psychosocial exposure was measured using the Job Content Questionnaire to assess risk among those with high job strain measures. Individual level occupational biomechanical exposures included the \%time spent $>30^{\circ}$ wrist extension, $\%$ time in $>30^{\circ}$ wrist flexion, total repetition rate, and the $\%$ time spent in forceful exertion ( $>1 \mathrm{~kg}$-pinch; $>4 \mathrm{~kg}$-grip). A subcohort of 1091 participants had both psychosocial and biomechanical exposure data. Adjusted hazard ratios were estimated using Cox proportional hazards models.

Results After adjustment for gender, age and BMI in the subcohort, high job strain (HR $=1.40 ; 95 \% \mathrm{CI}: 0.86-2.28)$ and high psychological demand ( $\mathrm{HR}=1.25 ; 95 \% \mathrm{CI}: 0.79-1.98)$ showed statistically non-significant elevation in risk of CTS, and high decision latitude ( $\mathrm{HR}=0.70$; 95\% CI:0.44-1.13) showed nonsignificant decrease in risk. When the same models were adjusted for biomechanical exposures, confounding was not evident; the primary exposure effect estimates changed between $1-7 \%$ for high job strain $(\mathrm{HR}=1.30 ; 95 \% \mathrm{CI}: 0.81-2.17)$, high psychological demand ( $\mathrm{HR}=1.17 ; 95 \% \mathrm{CI}: 0.74-1.83)$, and high decision latitude (HR=0.71; 95\% CI:0.43-1.18).

Conclusions For this sub-cohort analysis, adjustment for biomechanical exposures did not alter the associations between workplace psychosocial factors and incident CTS. The findings suggest that workplace psychosocial risk is independent of workplace biomechanical risk.

\section{OCCUPATIONAL EXPOSURE TO BENZENE AND RISK OF NON-HODGKIN LYMPHOMA IN A POPULATION-BASED COHORT STUDY OF CHINESE WOMEN IN SHANGHAI}

${ }^{1}$ Bryan Bassig, ${ }^{1}$ Melissa C Friesen, ${ }^{2}$ Roel Vermeulen, ${ }^{3} \mathrm{Xiao}-\mathrm{Ou}$ Shu, ${ }^{1}$ Mark Purdue, ${ }^{1}$ Patricia Stewart, ${ }^{4}$ Wei Lu, ${ }^{5}$ Yong-Bin Xiang, ${ }^{6}$ Wong-Ho Chow, ${ }^{7}$ Tongzhang Zheng, ${ }^{1}$ Bu-Tian Ji, ${ }^{3}$ Gong Yang, ${ }^{8}$ Martha Linet, ${ }^{1}$ Wei Hu, ${ }^{7}$ Heping Zhang, ${ }^{3}$ Wei Zheng, ${ }^{5} \mathrm{Yu}$-Tang Gao, 'Nathaniel Rothman, 'Qing Lan. 'Occupational and Environmental Epidemiology Branch, Division of Cancer Epidemiology and Genetics, National Cancer Institute, Bethesda, MD, USA; ${ }^{2}$ Institute for Risk Assessment Sciences, University of Utrecht, Utrecht, The Netherlands; ${ }^{3}$ Division of Epidemiology, Department of Medicine, Vanderbilt Epidemiology Center, Vanderbilt University School of Medicine, Nashville, TN, USA; ${ }^{4}$ Shanghai Municipal Center for Disease Control, Shanghai, China; ${ }^{5}$ Department of Epidemiology, Shanghai Cancer Institute, Shanghai, China; ${ }^{6}$ Department of Epidemiology, The University of Texas MD Anderson Cancer Center, Houston, TX, USA; ${ }^{7}$ Yale University School of Public Health, New Haven, CT, USA; ${ }^{8}$ Radiation Epidemiology Branch, Division of Cancer Epidemiology and Genetics, National Cancer Institute, Bethesda, MD, USA

\subsection{6/oemed-2014-102362.125}

Objectives The association between benzene exposure and non-Hodgkin lymphoma (NHL) has been the subject of debate, and an IARC working group recently concluded for the first time that there is now limited evidence to support this association in humans. We evaluated the relationship between occupational benzene exposure and NHL risk among 73087 women in a population-based cohort study of women in Shanghai.

Method Benzene exposure estimates were derived using a previously developed exposure assessment framework that combined ordinal job-exposure matrix intensity ratings with quantitative benzene exposure measurements from an inspection database of Shanghai factories collected between 1954-2000. Associations between benzene exposure metrics and NHL $(n=102)$ were assessed using Cox proportional hazard models, with study follow-up occurring from 1997-2009. 
Results Women ever exposed to benzene had a significantly elevated risk of NHL (Hazard Ratio $(\mathrm{HR})=1.87,95 \% \mathrm{CI}=$ 1.19-2.96). Compared to unexposed women, significant trends in NHL risk were observed for increasing years of benzene exposure $\left(\mathrm{p}_{\text {trend }}=0.009\right.$ ) and increasing cumulative exposure levels $\left(\mathrm{p}_{\text {trend }}=0.01\right)$, with women in the highest duration and cumulative exposure tertiles having a significantly elevated association with NHL $(\mathrm{HR}=2.07,95 \% \mathrm{CI}=1.07-4.01$ and $\mathrm{HR}=$ $2.16,95 \%$ CI $=1.17-3.98$, respectively).

Conclusions Our study is the first to our knowledge to evaluate this association in the context of a population-based prospective cohort of all women with diverse occupational histories. Our findings add to the evidence that benzene is associated with risk of NHL.

\section{A STUDY OF RADIOGRAPHIC ABNORMALITIES IN MINNESOTA TACONITE WORKERS}

Jeff Mandel, Christine Lambert, Bruce Alexander, Richard MacLehose, Gurumurthy Ramachandran. University of Minnesota, Minneapolis, USA

\subsection{6/oemed-2014-102362.126}

Objectives A limited number of studies have been conducted in the taconite industry, typically without detailed exe posure information. We assessed occupational exposure to elongate mineral particles (EMP) in association with chest $x$-ray findings in a cross-sectional screening of 1188 current and former taconite workers.

Method Exposure was determined by a combination of some 1300 onsite measures taken by researchers and historical measures taken by mining companies. The NIOSH 7400 measurement method (PCM) was used to count EMP. Twenty-eight similarly exposed groupings (SEG) were developed and contained all jobs. Each SEG had multiple EMP measures as an anchoring point for historical exposure estimates. Work histories were standardised, mapped to SEGs and used to estimate exposures for each worker in each SEG. Chest x-rays were obtained using ILO methods, with two independent radiologists. Prevalence odds ratios were estimated by logistic regression for work duration and cumulative EMP exposure, adjusting for age, gender, BMI and occupations with high potential for asbestos.

Results Pleural findings occurred in $16.8 \%$ by consensus. Abnormalities were associated with duration of employment in the taconite industry for those working 21 years or more $(\mathrm{OR}=1.59,95 \% \mathrm{CI}=1.06-2.40)$. Pleural abnormalities were also associted with cumulative exposure to EMP for those cumulatively exposed to $1.16 \mathrm{EMP} / \mathrm{cc}$-years or more $(\mathrm{OR}=1.93,95 \%$ $\mathrm{CI}=1.32-2.83)$. Associations between parenchymal abnormalities and exposure were not observed.

Conclusions This study suggests that pleural abnormalities are related to duration of employment and to EMP exposure in taconite miners. Due to the measurement method, the specific type of EMP could not be described further.

\section{THE CONTRIBUTION OF OCCUPATIONAL STUDIES TO THE IDENTIFICATION OF CARCINOGENS IN THE IARC MONOGRAPHS AND HOW TO FURTHER IMPROVE IT}

Dana Loomis, Neela Guha, Kurt Straif. IARC, Lyon, France

10.1136/oemed-2014-102362.127

Objectives To discuss the important role of occupational studies in identifying carcinogens and suggest how it could be still greater.
Method The contribution of occupational studies to the IARC monographs is reviewed.

Results Occupational epidemiology has made important contributions beyond developing knowledge to protect workers' health, notably in identifying carcinogens of concern for the general population. The IARC Monographs have evaluated many carcinogens for which occupational studies have provided key evidence. The recent classifications of diesel engine exhaust, trichloroethylene and polychlorinated biphenyls (PCBs) as human carcinogens, which depended heavily on data from occupational studies, are illustrative. In the evaluation of PCBs, for example, occupational cohort studies showing an exposure-related increase in the risk of malignant melanoma were pivotal for the conclusion of sufficient evidence of carcinogenicity. Despite such noteworthy contributions, the number of occupational studies that are ultimately informative tends to be relatively small relative to the number reviewed. The most informative studies tend to have common features, including clear reporting of methods and results, well-defined outcomes, quantitative estimates of exposure, adequate control of major confounders, and state of the art analytical methods, often with internal analyses of exposure-response. In contrast, studies that are too broadly focused and those with crude classifications of exposure or outcome, analyses by external comparisons alone or poor reporting of the methods and results are often less informative in the final evaluation.

Conclusions While occupational studies are important for carcinogen identification, their relevance could be further enhanced with improvements in study design, methods and reporting.

\section{YEARS (1989-2008) OF DIRECT COSTS ASSOCIATED WITH FALLS FROM HEIGHT AMONG UNION CARPENTERS, WASHINGTON STATE, USA}

${ }^{1}$ Hester Lipscomb, ${ }^{1}$ Ashley Schoenfisch, ${ }^{2}$ Wilfrid Cameron, ${ }^{3}$ Kristen Kucera, ${ }^{4}$ Darrin Adams, ${ }^{4}$ Barbara Silverstein. ${ }^{1}$ Duke University, Durham, NC, USA; ${ }^{2}$ Strategic Solutions for Safety, Health and Environment, Seattle, Washington, USA; ${ }^{3}$ University of NC, Chapel Hill, NC, USA; ${ }^{4}$ SHARP, Department of Labor and Industries, Olympia, Washington, USA

\subsection{6/oemed-2014-102362.128}

Objectives To examine trends in workers' compensation payments for falls from height (FFH) among a large cohort of carpenters over a 20-year period (1989-2008). Cost data provide an important metric reflecting frequency of falls and severity of associated injuries.

Method Using combined administrative data we evaluated workers' compensation (WC) payments associated with FFH among a large ( $\mathrm{n}=24$ 830) 20-year cohort (1989-2008) of union carpenters in Washington State. Mean payments, costs rates and adjusted rate ratios based on hours worked were calculated using negative binomial regression to evaluate cost patterns based on age, union tenure, type of carpentry work and calendar time after adjusting and discounting to 2011 dollar values.

Results FFH accounted for $\$ 66.6$ million in WC payments (a burden of $\$ 0.35$ per hour worked) over the 20-year period. FFH were responsible for $5.5 \%$ of injuries but $15.1 \%$ of costs. Marked cost declines were observed over time, but not in a monotonic fashion. Reductions were more pronounced for indemnity than for medical care. Mean costs per fall were unchanged in the latter years of observation from those observed 1995-1996. Individuals performing millwrighting or drywall installation had cost rates over twice as high as commercial construction. Mean costs were 2.3 times greater among carpenters 